\title{
Reverse Nearest Neighbors Search in Ad Hoc Subspaces
}

\author{
Man Lung Yiu and Nikos Mamoulis
}

\begin{abstract}
Given an object $q$, modeled by a multidimensional point, a reverse nearest neighbors (RNN) query returns the set of objects in the database that have $q$ as their nearest neighbor. In this paper, we study an interesting generalization of the RNN query, where not all dimensions are considered, but only an ad hoc subset thereof. The rationale is that 1) the dimensionality might be too high for the result of a regular RNN query to be useful, 2) missing values may implicitly define a meaningful subspace for RNN retrieval, and 3) analysts may be interested in the query results only for a set of (ad hoc) problem dimensions (i.e., object attributes). We consider a suitable storage scheme and develop appropriate algorithms for projected RNN queries, without relying on multidimensional indexes. Given the significant cost difference between random and sequential data accesses, our algorithms are based on applying sequential accesses only on the projected atomic values of the data at each dimension, to progressively derive a set of RNN candidates. Whether these candidates are actual RNN results is then validated via an optimized refinement step. In addition, we study variants of the projected RNN problem, including R $k$ NN search, bichromatic RNN, and RNN retrieval for the case where sequential accesses are not possible. Our methods are experimentally evaluated with real and synthetic data.
\end{abstract}

Index Terms-Distributed databases, query processing, spatial databases.

\section{INTRODUCTION}

C ONSIDER a set $\mathcal{D}$ of objects that are modeled as points in a multidimensional space, defined by the domains of their various features. Given a query object $q$, a reverse nearest neighbor (RNN) query [19], [25], [29], [26], [5], [20], [24], [28], [31] retrieves all objects in $\mathcal{D}$ that have $q$ closer to them than any other object in $\mathcal{D}$ (according to a distance measure). RNN queries are used in a wide range of applications such as decision support, resource allocation, and profile-based marketing.

Assume, for example, that $\mathcal{D}$ is a set of films in a database (owned by a video rental shop) and that each dimension is the rating of the film based on its relevance to a different category (e.g., action, comedy, detective, horror, political, historical, etc.). The rating of a film at a particular dimension is determined by averaging the opinions of customers who have watched the film. Fig. 1 shows a few films as points in a multidimensional space, considering only two dimensions: action and comedy. In this space, $a$ and $e$ are the reverse nearest neighbors of $q$ (based on Euclidean distance); these two points have $q$ as their nearest neighbor $(\mathrm{NN})$. The query result could be used to recommend $q$ to customers who have watched $a$ or $e$ since they could be interested in $q$ as well. Note that the NN of $q$ (i.e., $b$ ) is not necessarily the $\mathrm{RNN}$ of $q$ (since $c$ is closer to $b$ ), thus NN and RNN queries are essentially two different problems. In addition, RNN queries can have multiple

- M.L. Yiu is with the Department of Computer Science, Aalborg University, DK-9220 Aalborg, Denmark. E-mail:mly@cs.aau.dk.

- N. Mamoulis is with the Department of Computer Science, University of Hong Kong, Pokfulam Road, Hong Kong. E-mail: nikos@cs.hku.hk.

Manuscript received 4 May 2006; revised 26 Sept. 2006; accepted 2 Oct. 2006; published online 18 Jan. 2007.

For information on obtaining reprints of this article, please send e-mail to: tkde@computer.org, and reference IEEECS Log Number TKDE-0230-0506. results, as opposed to $\mathrm{NN}$ queries which have exactly one result.

We have illustrated RNN queries based on only two dimensions; however, there may be a large number of dimensions, in general. According to [6], NN search (and RNN search, by extension) could be meaningless in highdimensional spaces, due to the well-known curse of dimensionality. This fact motivated database researchers to study range queries [23], clustering [3], and similarity search [17] in dimensional subspaces where they could be meaningful. The searched subspace is ad hoc and may vary between different similarity (and RNN) queries. For instance, assume that a new film is registered in the database and watched by some customer. The customer rates the film only based on three dimensions (e.g., action, detective, and political), while leaving the rest of the ratings blank. In this case, there is no other meaningful way to search for the RNN of the film, but using only these three dimensions. Gessert [14] and Ooi et al. [23] stress the need for queries in attribute subspaces, due to the existence of missing values. Such projected RNN queries could also be applied if some attributes of the query tuple are not relevant for search [14]. A data analyst could explicitly select an ad hoc-dimensional subspace to search which he thinks is interesting. This scenario is very common in business analysis tasks, which aggregate data based on ad hocdimensional subspaces in online analytical processing (OLAP) applications. Thus, we argue that projected NN and RNN queries in ad hoc-dimensional subspaces are as important as their counterparts that consider the fulldimensional space, especially in very high-dimensional data collections.

Surprisingly, in spite of the huge bibliography in OLAP [1], to our knowledge, there is no prior work on NN and RNN search in ad hoc-dimensional subspaces. Regarding 


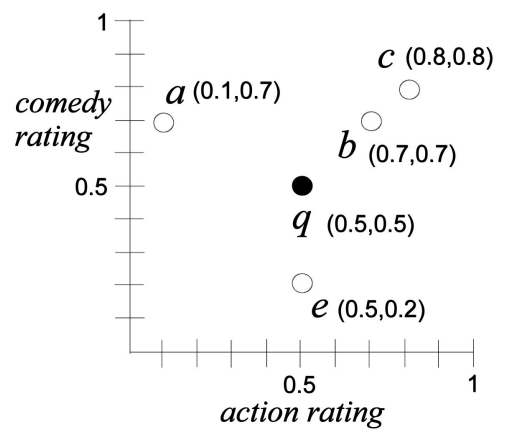

Fig. 1. Films rating database.

$\mathrm{NN}$ queries, we can attribute this lack of research to the fact that they can be straightforwardly converted to (and solved as) top- $k$ queries [11], [16], as we will discuss later (Section 3.2). However, RNN retrieval is more complex and there is no straightforward adaptation of existing work [19], [25], [29], [28] for the projected version of this problem.

In this paper, we fill this gap by proposing appropriate projected RNN evaluation techniques. Our solution is based on the decomposition storage model (DSM) [10]. A commercial product [2] and two prototype DBMS ([7] and [27]) have been developed based on DSM. These systems are most appropriate for business analysis operations (e.g., OLAP) in large, relatively static collections, where only a small number of attributes are relevant to each query. In DSM, a binary table is created for each attribute, storing for each original tuple, the ID of the tuple, and the value of the attribute in that tuple. The binary table can be sorted on attribute value and/or could be indexed by a $\mathrm{B}^{+}$-tree to facilitate search. As a result, only relevant tables need to be accessed for a query that relates to an ad hoc set of attributes/dimensions. Vertically fragmented data can be either centralized or distributed. In the distributed model, the binary tables are located at separate servers and remotely accessed by users operating client machines. The queried data are transferred in the form of a stream that stops when the whole result is transmitted or the server receives a termination message from user. A popular example of querying decomposed distributed data is combining object rankings from different sources [11], [16].

The main objective of our DSM-based RNN algorithms is to minimize the number of accessed tuples from the binary tables since they reflect I/O cost in the centralized model and communication cost in the distributed model. The minor objectives are to reduce computational time and memory usage. To our knowledge, ours is the only work that studies projected RNN queries by gracefully applying search on vertically decomposed data.

In addition to the more generic problem settings, we also study some interesting variants of RNN search. The first is the RkNN query, which retrieves the set of objects having the query $q$ in their $k \mathrm{NN}$ set. The second is the bichromatic RNN query [26], which takes as input a point $q$ from a data set $T$ (e.g., hotels) and finds the points in another data set $P$ which are closer to $q$ than any other point in $T$. Third, we study the case where the data are vertically decomposed and distributed to different servers that allow only random accesses. In such a case, for each object, it is essential to access at least one atomic value from a server before we can decide whether the object can be pruned from the set of RNN candidates. We develop methods that minimize the required number of accesses for deriving the RNN result.

The rest of the paper is organized as follows: Section 2 discusses related work. Section 3 defines the problem and outlines the RNN algorithmic framework. Sections 4 and 5 present our methodology. Section 6 discusses interesting RNN variants. Experimental results are presented in Section 7. Finally, Section 8 concludes the paper.

\section{Related Work \\ 2.1 Euclidean RNN Search}

The basic idea of early RNN algorithms [19], [29] is to precompute the $\mathrm{NN}$ distance for each data point $p$. An index is built on the points with their NN distances so that RNN results can be retrieved fast. This approach has an expensive update cost for dynamic data sets. In addition, since we want to support RNN search in ad hoc sets of dimensions, it is infeasible to materialize the RNN for all points in all possible subspaces.

Thus, research shifted toward methods that do not rely on precomputation. These algorithms follow a filter-refinement framework. In the filter step, a set of candidate RNN points (i.e., a superset of the actual result) is retrieved. During the refinement (verification) step, a range query is applied around each candidate $p$ to verify whether the query point $q$ is closer to $p$ than any other point in the database. If so, $p$ is reported as an RNN of $q$. The algorithms of [25], [28] apply on R-trees and rely on geometric properties of the Euclidean distance. Stanoi et al. [25] divide the (2D) data space around $q$ into six regions. It can be proved for each region that either 1) the region does not have any RNN or 2) the RNN of $q$ in the region is exactly the $\mathrm{NN}$ of $q$, when only the points in the region are considered. Thus, in the filter step, six constrained NN queries [13] are issued to find the nearest neighbor of $q$ at each region.

Tao et al. [28] propose a more efficient geometric solution (TPL) for the filter step. An incremental NN (INN) algorithm is employed to retrieve candidate points continuously from the R-tree that indexes the data points. The original INN algorithm [18] first inserts all root entries of the tree into a priority queue based on their distance from $q$. The nearest entry to $q$ is retrieved from the queue; if it is a leaf entry, the corresponding object is reported as the next nearest neighbor. If it is a directory entry, the corresponding node is visited and its entries are inserted into the queue. TPL when visiting a node, before inserting its entries into the priority queue, trims their minimum bounding rectangles (MBRs) using the already computed RNN candidates to smaller rectangles by pruning the areas of them which may not contain RNN results. Fig. 2 shows an example after two candidate points $a$ and $b$ are discovered. Assume that point $a$ is retrieved first and let $M$ be the MBR of a node not accessed yet. The perpendicular bisector $\perp(a, q)$ of points $a$ and $q$ partitions the data space into two regions: halfplane $\perp_{q}(a, q)$ containing points closer to $q$ than $a$ and halfplane $\perp_{a}(a, q)$ containing points closer to $a$ than $q$. Note that 


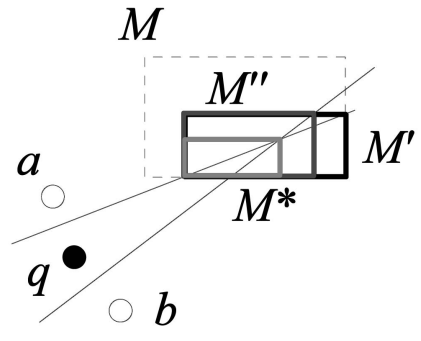

Fig. 2. Example of TPL.

$\perp_{a}(a, q)$ cannot contain any RNN results; thus, we only need to consider $M \cap \perp_{q}(a, q)$ in subsequent search.

Since the exact $M \cap \perp_{q}(a, q)$ may have complex representation (the MBR could be trimmed by multiple bisectors and could be of high dimensionality), [28] suggested approximating the trimmed region by its MBR. Consecutive clipping of an MBR is applied if there are multiple candidate RNNs intersecting it. For instance, $M$ is clipped first to $M^{\prime}$ and then to $M^{\prime \prime}$, after considering $\perp(a, q)$ and $\perp(b, q)$ in this order. Although this clipping technique has low computational cost, it may not result in the smallest possible MBR. Observe that the best MBR enclosing the nonpruned region in $M$ is $M^{*}$ instead of $M^{\prime \prime}$.

RNN search is a popular problem, many variants of which have been proposed. Singh et al. [24] propose an approximate algorithm which cannot guarantee the discovery of all results. Stanoi et al. [26] focus on bichromatic RNN queries. Benetis et al. [5] investigate RNN queries on spatiotemporal data. Korn et al. [20] examine aggregate RNN queries which return an aggregate of the RNN set, on 1D data streams. Finally, [31] studies RNN queries on graphs where the distance between two objects is determined by their shortest path.

The main defect of existing RNN methods is that they rely either on materialization of results or on multidimensional indexes (e.g., R-trees); thus, they are not effective in solving the projected RNN problem stated in Section 1. The data set may have a large number of dimensions and the user could select only an arbitrary, small, interesting subset of them, which is different from query to query. Construction and maintenance of numerous (i.e., $2^{d}-1$ for $d$ dimensions) specialized indexes for all attribute subsets is too expensive (or infeasible for vertically fragmented distributed data). Besides, existing techniques [25], [28] rely on geometric properties specific for the Euclidean distance, and they cannot be applied for other distance measures (e.g., Manhattan distance).

\subsection{Top- $k$ Queries}

Our problem is closely related to top- $k$ queries. Given a set of objects and a number of rankings for these objects according to different criteria, a top- $k$ query retrieves the $k$ objects with the highest combined score. Assume, for example, that we wish to retrieve the restaurants in a city in decreasing order of their aggregate scores with respect to how cheap they are, their quality, and their closeness to our hotel. If three separate services can incrementally provide ranked lists of the restaurants based on their scores in each of the query components, the problem is to identify the $k$ restaurants with the best combined (e.g., average) score.

There are two types of primitive operations used by top- $k$ algorithms: random accesses and sorted accesses. A random access retrieves the value of a particular object (given its ID) for a particular dimension (i.e., attribute). The alternative (sorted accesses) is to retrieve objects from each ranking sequentially, in decreasing order of their scores.

The two main top- $k$ retrieval paradigms [11] are: the Threshold Algorithm (TA), which applies both sequential and random accesses, and No Random Accesses (NRA), which applies only sorted accesses. They share the following common points. Objects are retrieved from different sources by sorted accesses. A threshold $T$ is defined by aggregating the latest values seen by sorted accesses in all dimensions. The algorithm terminates when the $k$ th best score is higher than $T$ in the worst case.

Whenever TA sees an object by a sorted access, the values of the object in other dimensions are retrieved by using random accesses and its overall score is computed. The top- $k$ score is updated if necessary. The advantage of TA is that it requires minimal memory for maintaining top- $k$ objects. NRA only applies sorted accesses. Objects which have been seen in some ranking list are organized based on their overall score in the worst case (assuming minimal values for dimensions where the object has not been seen). Since information for all objects seen in some ranking are stored in memory, NRA has large memory requirements.

When we are looking for the $\mathrm{NN}$ of a point $q$ whose dimensional values have been decomposed to binary tables based on the DSM, we can apply a top- $k$ algorithm after retrieving the tuples from each binary table $A_{i}$ (corresponding to the $i$ th dimension) in increasing order of their absolute difference from $q_{i}$ (the value of $q$ in dimension $i$ ). In Section 3.2, we elaborate more on the relationship between top- $k$ queries and projected NN (and RNN) search.

\section{3 a Framework for RNN Search}

In this section, we set up the problem studied in this paper by proposing a storage model based on DSM and a framework for processing projected $\mathrm{NN}$ and $\mathrm{RNN}$ queries on this model.

\subsection{Problem Setting}

We consider a set $\mathcal{D}$ of $d$-dimensional points. $\mathcal{D}$ is stored in $d$ binary tables, one for each dimension. Table $A_{i}$ stores the IDs of all points in $\mathcal{D}$ and their values in the $i$ th dimension. The tables may be stored centrally or distributed to different servers. Let $p_{i}$ be the value of the point $p$ at dimension $i$. Given a value $q_{i}$, for all points $p$ satisfying $p_{i} \geq q_{i}\left(p_{i}<q_{i}\right)$, their values in the $i$ th dimension can be retrieved in ascending (descending) order by searching $A_{i}$ for $q_{i}$ and accessing the remainder of the table forward (backward) sequentially. Search can be facilitated by sparse $\mathrm{B}^{+}$-trees, built on top of the binary tables. We denote by $A_{i}^{+}\left(A_{i}^{-}\right)$a (virtual) table containing the values in $A_{i}$ greater (smaller) than $q_{i}$ in ascending (descending) order. Conceptually, we open two streams for each dimension involved in the query 
that return the smaller and greater values than $q_{i}$ in order by performing only sequential accesses.

We emphasize that only the set of query dimensions (instead of all dimensions) is considered during query processing. In the rest of the paper, we use $d$ to denote the number of query dimensions (not the data dimensionality). Our goal is to solve RNN queries based on the above data model. Definition 1 states the result set of an RNN query. Unless otherwise stated, we consider Euclidean distance as the dissimilarity function $\operatorname{dist}()$. We shall discuss other distance functions in Section 4.2.

Definition 1. Given a query point $q$ and a data set $\mathcal{D}$, a RNN query retrieves the set

$$
R N N(q)=\{p \in \mathcal{D} \mid \operatorname{dist}(p, q)<N N \operatorname{dist}(p, \mathcal{D})\},
$$

where $N N \operatorname{dist}(p, \mathcal{D})$ denotes the $N N$ distance of $p$ in $\mathcal{D}$.

\subsection{Incremental Nearest Neighbor Search}

In this section, we show how to adapt the NRA top- $k$ algorithm [11] for incremental retrieval of projected NN from our storage scheme. The proposed projected NN algorithm is extended to solve projected RNN queries in Section 3.3.

For each dimension $i$, tuples greater (smaller) than $q_{i}$ are retrieved from table $A_{i}^{+}\left(A_{i}^{-}\right)$, sequentially. We use $v\left(A_{i}^{+}\right)$ and $v\left(A_{i}^{-}\right)$to denote the last values seen from $A_{i}^{+}$and $A_{i}^{-}$, respectively. The value $p_{i}$ for a particular point $p$ is either in $A_{i}^{+}$or in $A_{i}^{-}$. Points which have been seen in some (but not all) dimensions are indexed in memory using a hash table. Let $\Lambda(p)$ be the set of dimensions where point $p$ has been seen. Considering Euclidean distance, we can compute the minimum possible distance of $p$ from $q$ as follows:

$$
\begin{aligned}
& \text { mindist }(q, p) \\
& =\sqrt{\sum_{i \in \Lambda(p)}\left|p_{i}-q_{i}\right|^{2}+\sum_{i \notin \Lambda(p)}\left(\min \left\{v\left(A_{i}^{+}\right)-q_{i}, q_{i}-v\left(A_{i}^{-}\right)\right\}\right)^{2}}
\end{aligned}
$$

since, in the best case, $p_{i}$ is equal to the closest of $v\left(A_{i}^{+}\right)$ and $v\left(A_{i}^{-}\right)$to $q_{i}$ in all dimensions $i$ where $p_{i}$ has not been seen yet. $^{1}$

Points which have been seen in all dimensions are removed from the hash table and inserted into a min-heap. Let $p_{t o p}$ be the top object in this heap. If $\operatorname{dist}\left(q, p_{t o p}\right)$ is smaller than mindist $(q, p)$ for all other points (including completely unseen points) $p \neq p_{\text {top }}$, then $p_{\text {top }}$ is output as the next NN. In this way, all NNs are (incrementally) output or the user may opt to terminate search after a satisfactory set of NN has been output.

\subsection{A Framework for RNN Search}

As discussed in Section 2.1, RNN algorithms operate in two steps: 1) The filter step retrieves a candidate set which contains all the actual results and 2) the verification step eliminates false hits and reports the actual RNNs. This framework allows us to consider filter algorithms and verification algorithms independently. In this section, we

1. If, for some dimension $i, A_{i}^{+}$is exhausted, then term $v\left(A_{i}^{+}\right)-q_{i}$ is removed. Similarly, if $A_{i}^{-}$is exhausted, term $q_{i}-v\left(A_{i}^{-}\right)$is removed.

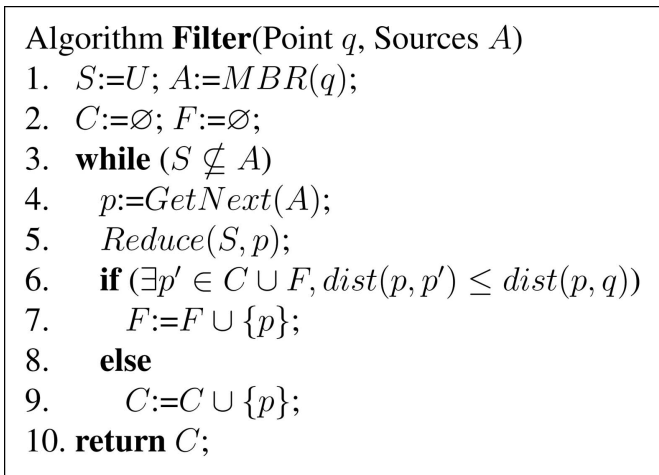

Fig. 3. The filter algorithm.

focus on the filter step because it dominates the overall cost (as verified in our experiments). Verification algorithms will be discussed in detail in Section 5 .

Fig. 3 shows a high-level pseudocode describing the framework of RNN algorithms that operate on decomposed data. In simple words, the RNN algorithms expand the space around $q$, discovering RNN candidates and, at the same time, constraining the additional space that needs to be searched by exploiting the locations of discovered points. $S$ denotes the MBR of the space that potentially contains RNNs of the query point $q$, not found yet. Initially, it is set to the MBR of the universe $U$ since there is no information about the location of RNNs before search.

Let $v\left(A_{i}^{+}\right)$and $v\left(A_{i}^{-}\right)$be the last values seen on files $A_{i}^{+}$ and $A_{i}^{-}$, respectively, by sorted accesses. The accessed space $A=\left(\left[v\left(A_{1}^{-}\right), v\left(A_{1}^{+}\right)\right],\left[v\left(A_{2}^{-}\right), v\left(A_{2}^{+}\right)\right], \cdots,\left[v\left(A_{d}^{-}\right), v\left(A_{d}^{+}\right)\right]\right)$, is defined by the minimum bounding rectangle (MBR) of the values seen at all binary tables. First, we assign the MBR of $q$ to $A$, indicating that sorted accesses along the $i$ th dimension start bidirectionally from the value $q_{i}$. Let $C$ be the candidate set and $F$ be the set of points (false hits) that have been seen in all dimensions, but are not RNNs. Pruned points are maintained in $F$ in order to assist early identification of whether some candidates are false hits (see Line 6 of the algorithm). Initially, both $C$ and $F$ are set to empty. We will illustrate the semantics of $C$ and $F$ shortly.

The filter algorithm has two core operations, GetNext and Reduce. Here, we only state their specifications. Their concrete implementations will be studied in Section 4 . The function $G e t N \operatorname{ext}(A)$ probes the set of binary tables $A$ (e.g., in a round-robin fashion) and then returns a complete point $p$ whose values in all dimensions have been seen. The function $\operatorname{Reduce}(S, p)$ uses $p$ to reduce the search space $S$.

By Definition 1, if a point $p$ is nearer to some other point $p^{\prime}$ than $q$, then $p$ cannot be an RNN of $q$. In this case, $p$ is said to be pruned by $p^{\prime}$. At Line 6 of the algorithm, we check whether $p$ can be pruned by some other points in $C$ or $F$. If so, $p$ is pruned and then added to $F$. Otherwise, $p$ is added to the candidate set $C$ because it is a potential result. The filter step terminates as soon as the space to be searched, $S$, is completely covered by the accessed space $A$ (i.e., no more candidates can be discovered). Note that if $S$ is covered by $A$ in some dimensions and directions, the corresponding tables are pruned from search. Formally, for each dimension $i$, let $\left[S_{i}^{-}, S_{i}^{+}\right]$be the projection of $S$ in $i$. If 
$v\left(A_{i}^{-}\right)<S_{i}^{-}$, then stream $A_{i}^{-}$is pruned. Similarly, if $v\left(A_{i}^{+}\right)>S_{i}^{+}$, then stream $A_{i}^{+}$is pruned.

\section{FiLter Algorithms}

In this section, we propose filter algorithms for RNN search. Section 4.1 discusses an adaptation of the TPL algorithm [28] on our data model. Section 4.2 proposes a carefully designed and efficient RNN algorithm. Section 4.3 presents an on-the-fly progress indicator for the filter step. The algorithms follow the framework of Fig. 3; thus, we confine our discussion on the implementation of GetNext and Reduce operations.

\subsection{The TPL Filter}

The TPL filter algorithm adapts the access pattern and pruning techniques of the TPL algorithm [28], however, without relying on R-trees. The GetNext function of TPL returns the next $\mathrm{NN}$ of $q$ by applying the incremental algorithm described in Section 3.2. The Reduce function shrinks the search space $S$ by applying the clipping method of [28] directly on $S$. Let $p$ be the next NN of $q$. Formally, $\operatorname{Reduce}(S, p)$ returns the MBR enclosing $S \cap \perp_{q}(p, q)$.

The main disadvantage of the TPL filter is that MBR clipping introduces more dead space than necessary (as discussed in Section 2.1). Thus, it does not prune the search space effectively, increasing the number of accesses. A minor disadvantage is that it employs incremental NN search. In Section 4.2, we show that we can take advantage of points seen in all dimensions as soon as they are identified, no matter whether they are the next NN of $q$ or not.

\subsection{The Greedy Filter}

The Greedy filter algorithm is a carefully designed RNN algorithm on our data model which does not share the drawbacks of the TPL filter algorithm. The GetNext function of our algorithm is not based on incremental NN search. Instead, we modify the process of Section 3.2 to immediately return a point as soon as it has been seen in all dimensions. The rationale is that complete points seen earlier than the next NN may shrink the search space fast, allowing earlier termination of the filter step.

The Greedy filter algorithm also applies an improved method for reducing the search space $S$. The idea is based on the progressive computation of the Voronoi cell $V(q)$ of $q$. The Voronoi diagram [22] of a data set $\mathcal{D}$ partitions the space into a number of cells (polygons), one for each point in $\mathcal{D}$, such that, for every $p \in \mathcal{D}$, every point inside the Voronoi cell $V(p)$ (of $p$ ) is closer to $p$ than any other point in $\mathcal{D}$. Since the Voronoi cell of a point $p$ must be adjacent to that of its NN, the RNN set of $q$ is a subset of the points $p$ for which $V(q)$ and $V(p)$ are adjacent.

Computation and maintenance of Voronoi diagrams for each combination of dimensions and any distance measure is infeasible. In addition, past work for dynamic Voronoi cell computation of an arbitrary point exactly [32] or approximately [26] is based on intersections of bisectors, which is computationally expensive, especially for high dimensionality and arbitrary (i.e., non-Euclidean) distance measures. Besides, [4] proposes an offline method for computing an approximation of $V(q)$ with asymptotic

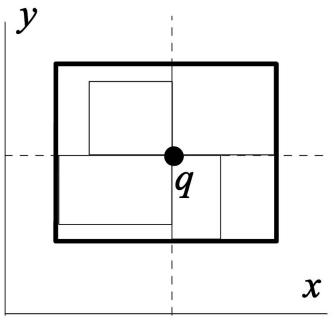

(a)

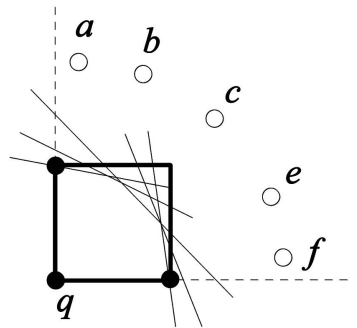

(b)
Fig. 4. Voronoi cell approximation. (a) Global search space. (b) Local search space.

bounds on approximation quality and space complexity. Such a method requires examining many points in the data set and it cannot be adapted to solve our problem where the points are discovered online.

The Greedy filter algorithm computes a progressively more refined approximation of $V(q)$ (and the corresponding neighboring points of $q$ that are candidate RNN results) while retrieving points. It can be easily shown that the MBR of $V(q)$ is the minimal possible space $S$ to be searched during the filter step. Let $W$ be a set of known (i.e., retrieved) points around $q$. Based on $W$, we can compute an approximation $V_{W}(q)$ of $V(q)$ by taking the intersection of all halfplanes $\bigcap_{p \in W} \perp_{q}(p, q)$. Halfplane intersection (for the $L_{2}$ norm) is both computationally expensive and space consuming. According to [22], each incremental computation requires $O\left(|W|^{\lceil d / 2\rceil}\right)$ time and $O\left(d|W|^{\lceil d / 2\rceil}\right)$ space (vertices of the resulting Voronoi cell). In addition, computation of halfplanes is far more complex for distance metrics other than $L_{2}$. Finally, halfplane intersection cannot be directly applied for RkNN search, which will be discussed in Section 6. We observe that setting the search space $S$ to any superset of $V_{W}(q)$ guarantees that no results outside the accessed space $A$ will be missed, thus exact computation of $V_{W}(q)$ may not be necessary for RNN retrieval. Next, we discuss two methods that compute conservative approximations of $V_{W}(q)$ that do not rely on halfplane intersection and can be computed for arbitrary $L_{p}$ distance norms.

\subsubsection{Approximation Using Intercepts}

Our first method approximates $V_{W}(q)$, dynamically and efficiently, as new points are retrieved. In addition, the approximated cell requires only bounded space, which is much smaller than the space required for representing the exact $V(q)$ in the worst case. Initially, we show how this method works with the Euclidean distance and then extend it for any $L_{p}$ distance norm.

First, we partition the search space around $q$ into $2^{d}$ quadrants, as shown in Fig. 4a. Consider the upper right quadrant in this example. Fig. $4 \mathrm{~b}$ illustrates how to derive the (local) search space for this quadrant. Suppose we have discovered five points, $a, b, c, e$, and $f$, there. For each point $p$ found $(p \in\{a, b, c, e, f\})$, we compute the intercepts of $\perp(p, q)$ with the axes of the quadrant. It turns out that it 


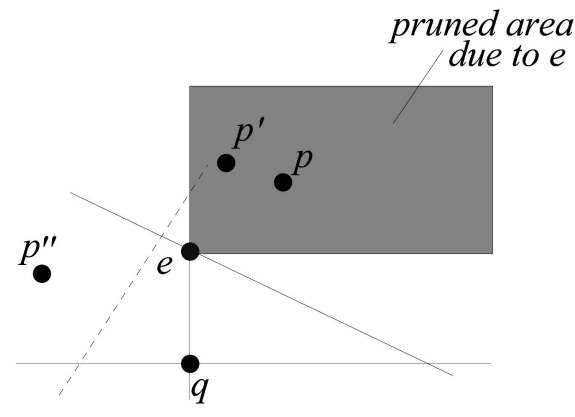

Fig. 5. Using intercepts to prune.

suffices to compute and maintain the intercept closest to $q$ for each dimension. Let $M$ be the MBR containing $q$ and these intercepts. Lemma 2 (using Lemma 1) guarantees that $M$ contains all potential RNNs in the quadrant. After $M$ has been computed for all quadrants, the (global) search space $S$ is taken as their MBR, as shown graphically in Fig. 4a.

Lemma 1. Consider the quadrant $Q$ with coordinates no smaller than $q$ in all dimensions. Let $p$ be a point in $Q$ and let $e$ be the intercept of $\perp(p, q)$ with some axis $r$, i.e., $e=\left(q_{1}, \ldots, q_{i-1}, c_{r}, q_{i+1}, \ldots, q_{d}\right)$. For any point $p^{\prime}$ for which $\forall i \in[1, d]: p_{i}^{\prime} \geq e_{i}$, we have $\operatorname{dist}\left(p^{\prime}, q\right) \geq \operatorname{dist}\left(p^{\prime}, p\right)$.

Proof. We first compare $\operatorname{dist}(e, q)$ and $\operatorname{dist}(e, p)$ with the corresponding distances $\operatorname{dist}\left(p^{\prime}, q\right)$ and $\operatorname{dist}\left(p^{\prime}, p\right)$ for every dimension individually. For any dimension $i$, let $\operatorname{diff}_{q}=$ $\left|p_{i}^{\prime}-q_{i}\right|-\left|e_{i}-q_{i}\right| \quad\left(\right.$ diff $_{q} \geq 0$ since $e_{i} \geq q_{i}$ and $\left.p_{i}^{\prime} \geq e_{i}\right)$. Similarly, let $\operatorname{diff}_{p}=\left|p_{i}^{\prime}-p_{i}\right|-\left|e_{i}-p_{i}\right|$. If $p_{i}^{\prime} \leq p_{i}$, then $\operatorname{diff}_{p} \leq 0$. If $p_{i}^{\prime} \geq p_{i}$, then $\operatorname{diff}_{p} \leq \operatorname{diff}_{q}$ since $q_{i} \leq p_{i} \leq p_{i}^{\prime}$. Thus, in any case, $\operatorname{diff}_{p} \leq \operatorname{diff}_{q}$. Since, in all dimensions, $p^{\prime}$ can only be closer to $p$ than $e$ is and $p^{\prime}$ can only be further from $q$ than $e$ is, and due to the monotonicity of the Euclidean distance (based on the atomic dimensional distances), we have $\operatorname{dist}\left(p^{\prime}, q\right) \geq \operatorname{dist}\left(p^{\prime}, p\right)$.

Lemma 2. Consider a quadrant $Q$ defined by $q$. Let $I$ be the set of the intercepts that are closest to $q$ for each dimension. Let $M$ be the $M B R$ defined by $q$ and these intercepts. $M$ encloses all $R N N s$ of $q$ in $Q$ that are located outside the accessed space $A$.

Fig. 5 shows graphically the area pruned by the intercept of $\perp(p, q)$ with the vertical quadrant axis. The intercept on the horizontal quadrant axis can similarly prune all points greater than or equal to it in all dimensions. Symmetrically, we can generalize the lemma for all quadrants of the search space. When multiple points exist in a quadrant, the nearest intercepts to $q$ dominate in pruning. Thus, Lemma 2 can be trivially proven.

We can prove versions of Lemmas 1 and 2 for any $L_{p}$ metric, since the basic proof (of Lemma 1) is based on the monotonicity property of Euclidean distance. An intercept coordinate $e=\left(q_{1}, \ldots, q_{i-1}, c_{r}, q_{i+1}, \ldots, q_{d}\right)$ for some axis $r$ of the halfplane between $q$ and a seen point $x$ can be easily computed from the equation $\operatorname{dist}(e, q)=\operatorname{dist}(e, x)$. Thus, our technique can be applied for any $L_{p}$ norm.

We stress that our Voronoi cell approximation technique is functionally different from the one in [26]. We use intercepts (based on any $L_{p}$ norm) to compute a rectangle that encloses $V(q)$, whereas [26] computes a more complex

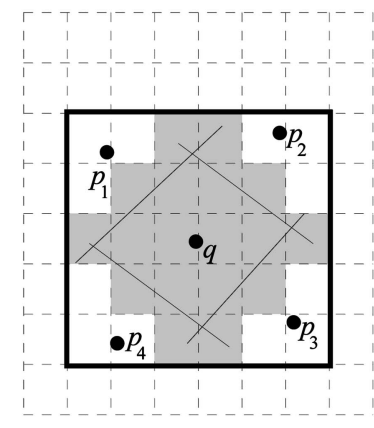

(a)

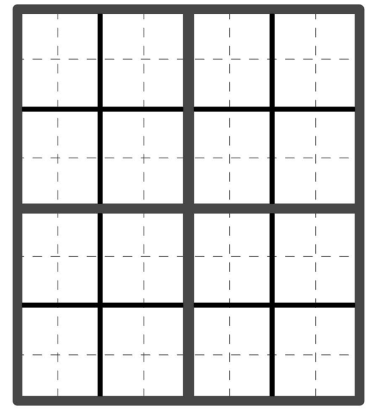

(b)
Fig. 6. Reducing search space using a grid. (a) A simple grid. (b) A hierarchical grid.

2D approximation of the cell. Thus, our method is applicable for any dimensionality (with significantly lower space requirements) and distance metric. Our approximation method is expected to outperform the TPL filter discussed in Section 4.1 since it optimally clips the quadrants containing points using information about these points. On the other hand, the TPL filter operates on the MBR of the whole search space $S$, which is harder to prune.

The only drawback of our technique is that each retrieved point is not utilized in pruning other quadrants except the one it resides in. For instance, in Fig. 5, point $p$ is used to prune the space only in the upper-right quadrant, whereas $\perp(p, q)$ could be used in combination with $\perp\left(p^{\prime \prime}, q\right)$ to prune $S$ also in the upper-left quadrant (note that $p^{\prime \prime}$ alone defines a greater $M$ there). In the next section, we propose another pruning technique that utilizes the effect of discovered points in neighboring quadrants.

\subsubsection{Approximation Using a Hierarchical Grid}

In this section, we propose a method that approximates the MBR that covers $V_{W}(q)$ with the help of a multidimensional grid. This approach has several advantages. First, it provides a guarantee on the quality of the approximation. Second, no memory is needed for storing the cells. Third, this technique can be used directly for other distance metrics. Initially, we assume that the Euclidean distance is used; later, we discuss other distance metrics.

Fig. 6a shows an exemplary $8 \times 8$ grid that partitions the search space $S$. Whenever a new point is retrieved by GetNext, we check (by the use of bisectors) whether a cell can be pruned by the points which have been seen in all dimensions. If not, the cell (shown in gray) is included in the revised search space $S^{\prime}$ for the next round (to be used for the next retrieved point). Instead of explicitly including all nonpruned cells in $S^{\prime}$, we consider the MBR of them (since the decomposed tables are essentially accessed until the MBR of $V(q)$ anyway). Thus, we need not explicitly maintain in memory any grid information. When the algorithm is invoked for the next point, the search space $S$ is smaller than before, thus the cells become smaller and the approximation quality improves incrementally.

Yet, the drawback of the above technique is that it requires high computational cost, especially in high-dimensional space, since a large number of cells must be checked. In 


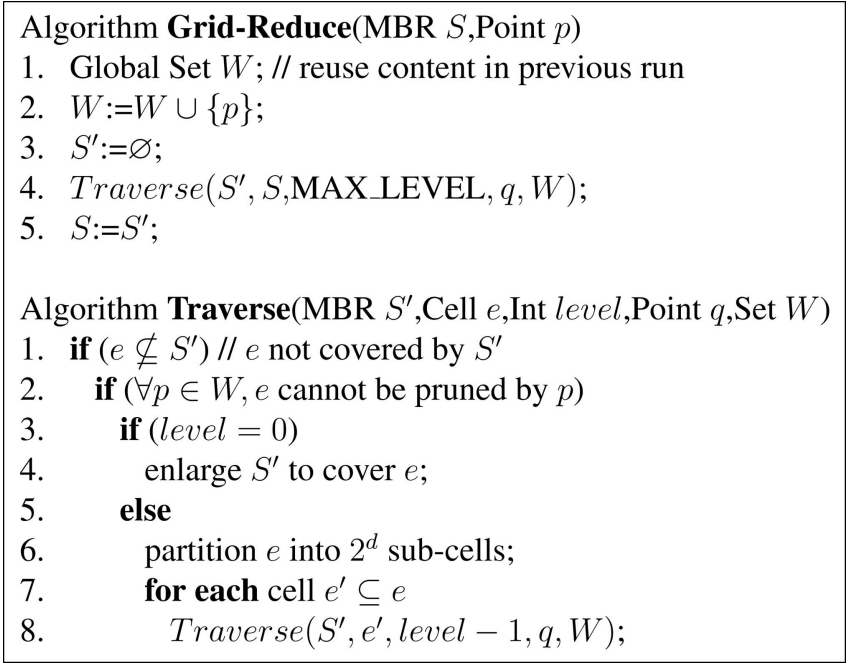

Fig. 7. Traversal algorithm for hierarchical grid.

order to reduce the CPU cost, we introduce a hierarchical grid, as shown in Fig. 6b, and employ branch-and-bound techniques to speed up computation. We first attempt to prune a high level cell. If pruning fails, then we partition it into smaller ones and apply the above procedure recursively. In Fig. $6 b$, the maximum recursion level is set to 3 . This parameter is a trade-off between the approximation quality and the computational cost.

Fig. 7 shows this hierarchical grid-based traversal algorithm for search space reduction. First, the newly discovered point $p$ is added to the set of points $W$, used for pruning (i.e., $W=C \cup F$ ). Then, we dynamically impose a hierarchical grid to the current search space $S$ and prune its cells hierarchically. $S^{\prime}$ denotes the output search space (MBR of cells that are not pruned). In the Traverse function, a cell $e$ is examined when 1) it is not covered by $S^{\prime}$ and 2) it cannot be pruned by any points in $W$. At recursion level 0 , pruning terminates and $S^{\prime}$ is enlarged to cover $e$. Note that the output $S^{\prime}$ of this algorithm is guaranteed to be no larger than $2 \bar{e}$ than the exact MBR of $V_{W}(q)$, in each dimension, where $\bar{e}$ is the length of a cell at the finest grid resolution. As a result, the proposed technique provides a good approximation guarantee.

The grid-based Greedy filter algorithm can be applied for other distance metrics by using alternative pruning methods for cells (i.e., not based on perpendicular bisectors), described by Lemma 3 (straightforwardly proven). In Fig. 8, the rectangle (i.e., cell) can be pruned since the maximum possible distance from a point in $M$ to $p(\operatorname{maxdist}(p, M))$ is smaller than the minimum distance from $M$ to $q(\operatorname{mindist}(q, M))$.

Lemma 3. Let $M$ be a rectangle. For any distance metric, if $\operatorname{maxdist}(p, M) \leq \operatorname{mindist}(q, M)$, then

$$
\forall p^{\prime} \in M, \operatorname{dist}\left(p, p^{\prime}\right) \leq \operatorname{dist}\left(q, p^{\prime}\right) .
$$

\subsection{Progress Indicator}

In the scenario where data are distributed over a slow network, communication cost is very expensive and it might be desirable for the user to have a progress indicator

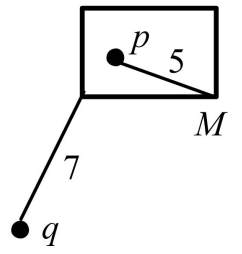

Fig. 8. Pruning for other distance metrics.

[21] for a long-running RNN query. During the execution of an RNN query, the expected remaining access cost in the filter step can be estimated as follows:

$$
\begin{aligned}
& \Psi(q, S, A)= \\
& |\mathcal{D}| \cdot \sum_{i \in[1, d]} \Gamma_{i}\left(x \in \mathcal{D},\left(S_{i}^{-} \leq x_{i} \leq A_{i}^{-}\right) \vee\left(A_{i}^{+} \leq x_{i} \leq S_{i}^{+}\right)\right),
\end{aligned}
$$

where $\Gamma_{i}$ captures the selectivity ${ }^{2}$ of query predicates on the $i$ th attribute.

Intuitively, the above formula expresses the number of accesses required to reach the search space $S$ from the accessed space $A$ (i.e., values within ranges $\left[S_{i}^{-}, A_{i}^{-}\right]$and $\left[A_{i}^{+}, S_{i}^{+}\right]$are expected to be accessed). However, the cost may be overestimated because future data accesses may shrink the search space $S$ and also reduce the remaining cost. To alleviate this problem, we now discuss how to derive a tighter search space. Recall that only completely seen points have been used to compute the search space $S$. In fact, we can guess the unseen values of partially seen points and then apply an RNN filter algorithm with those points for deriving a tighter search space $S^{*}$ from the existing $S$. For this, the (unseen) $i$ th attribute value of a point can be randomly generated (outside the range $\left[A_{i}^{-}, A_{i}^{+}\right]$) following the distribution of $\Gamma_{i}$. Finally, the estimated cost is averaged over multiple (e.g., 10) instances of $S^{*}$ in order to stabilize the randomness effect.

\section{Verification of Candidates}

In this section, we discuss whether the candidates obtained in the filter step are actual RNNs. In addition, we discuss early (progressive) computations of RNNs before the verification step. Finally, we show a method that minimizes $F$, i.e., the set of points that are not candidates, but they are used to prune $C$.

\subsection{Concurrent Verification}

The filter step terminates with a set $C$ of candidate points and a set $F$ of false hits; points that have been seen in all dimensions, but they are found not to be RNNs. Normally, each candidate $p \in C$ is verified by issuing a range search around $p$ with radius $\operatorname{dist}(q, p)$. If another point is found within this range, then $p$ is not an RNN of $q$; otherwise, it is returned. In order to reduce the number of range queries, we perform verification in two steps. First, we check each $p \in C$ whether they are closer to some other seen point in

2. The selectivity can be accurately computed if 1D histograms for each attribute are available. In case no histograms can be used, data distribution along each dimension can be estimated by maintaining moving averages of the values accessed. 


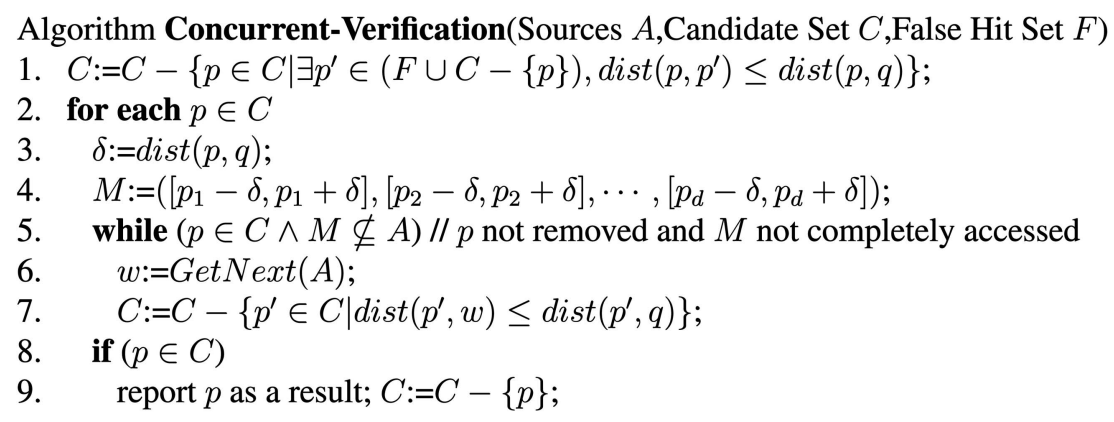

Fig. 9. Concurrent verification algorithm.

$C \cup F$ than to $q$. These candidates can be immediately eliminated.

The second step is to check the remaining candidates by range queries. Instead of issuing individual queries for each candidate, we perform a concurrent verification, which continues traversing the binary tables from the point where the filter algorithm has stopped, until all candidates have been verified. The overall verification algorithm is shown in Fig. 9. The main idea of the second step is to compute a rectangle $M$ for each candidate $p$ (based on $\operatorname{dist}(q, p)$ ), where its potential neighbors closer than $q$ may be contained. While accessing the binary tables in search of these points, each complete point $w$ is checked for whether it can prune any of the remaining candidates in $C$ (not only $p$ ). If $p$ cannot be pruned, then it is reported as a result.

\subsection{Progressive RNN Computation}

Our algorithmic framework allows early report of points that are definitely in the RNN set before the verification phase. A progressive report of results is very useful in practice since the user can examine early results while waiting for the complete response set.

Given a candidate point $p$, let $M(p)$ be the MBR enclosing the region with $p$ as the center and the range as $\operatorname{dist}(p, q)$. Formally, we have

$$
M(p)=\left(\left[p_{1}-\delta, p_{1}+\delta\right],\left[p_{2}-\delta, p_{2}+\delta\right], \cdots,\left[p_{d}-\delta, p_{d}+\delta\right]\right),
$$

where $\delta=\operatorname{dist}(p, q)$. During the filter step, if a candidate $p$ satisfies 1) $M(p) \subseteq A$ and 2)

$$
\forall p^{\prime} \in(C \cup F-\{p\}), \operatorname{dist}\left(p, p^{\prime}\right)>\operatorname{dist}(p, q),
$$

then $p$ can be immediately reported as a result. Fig. 10a shows an example, where $M(p)$ is enclosed in $A$ and does not contain any other point but $p$. Note that this is the first work to address progressive RNN computation without using materialized results.

\subsection{Reducing the Set of False Hits}

During the filter step, we maintain a potentially large set $F$ of points that are false hits, but may be used for candidate pruning. We can reduce this set by eliminating points that may not be used to prune any candidate. A point $p \in F$ can be discarded if 1) $p$ does not fall in the verification range of any existing candidate and 2) $\perp_{p}(q, p) \cap S \subseteq A . \perp_{p}(q, p)$ is the part of the data space containing points closer to $p$ than $q$. Only the points in this region can be pruned by $p$. If its intersection with the search space $S$ is already covered by the accessed space $A$, then any complete points found later cannot be pruned by the point $p$. Note that this condition can be generalized for arbitrary distance metrics by replacing $\perp_{p}(q, p)$ by the region closer to $p$ than to $q$. Fig. 10b illustrates an example, where a (noncandidate) point $p$ can be pruned from $F$.

\section{VARIANTS OF RNN SEARCH}

In this section, we discuss how our framework can be adapted for two common variants of basic RNN search: $\mathrm{R} k \mathrm{NN}$ queries and bichromatic RNN queries. We also investigate how RNN search can be deployed for the scenario where only random accesses (i.e., no sorted accesses) to data sources are allowed.

\subsection{R $k$ NN Search}

The filter and verification steps of RNN search in our framework can be easily adapted for the generalized problem of R $k$ NN search: Find all points $p$ such that $q$ belongs to the $k$-NN set of $p$. The TPL filter can be generalized for R $k \mathrm{NN}$ search if we select a $k$-subset $\left\{\theta_{1}, \theta_{2}, \cdots, \theta_{k}\right\}$ of the points in $C \cup F$. Let $\operatorname{clip}\left(S, q, \theta_{i}\right)$ be the MBR (in $S$ ) that may contain some points closer to the query point $q$ than the point $\theta_{i}$. Let $S^{\prime}$ be the MBR that encloses $\operatorname{clip}\left(S, q, \theta_{i}\right) \forall i \in[1, k]$. Observe that other RNN results cannot be outside $S^{\prime}$ because all such points are nearer to all $\theta_{1}, \theta_{2}, \cdots, \theta_{k}$ than to $q$. Therefore, $S^{\prime}$ becomes the new search space after a new point has been retrieved. Appropriate $k$-subsets of $C \cup F$ to be used for pruning can be selected using the heuristics of [28].

The Greedy filter can be adapted for R $k \mathrm{NN}$ search by considering the $k$ th closest intercept for each axis adjacent

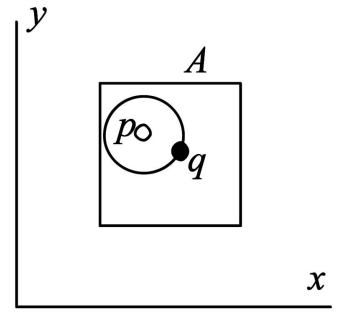

(a)

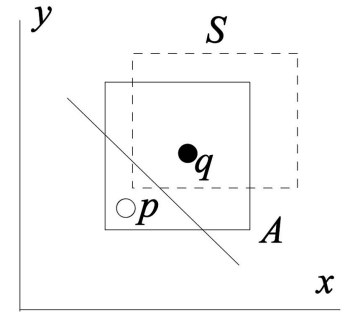

(b)
Fig. 10. Optimizing the filter step. (a) Progressive verification. (b) Reducing the refinement set. 


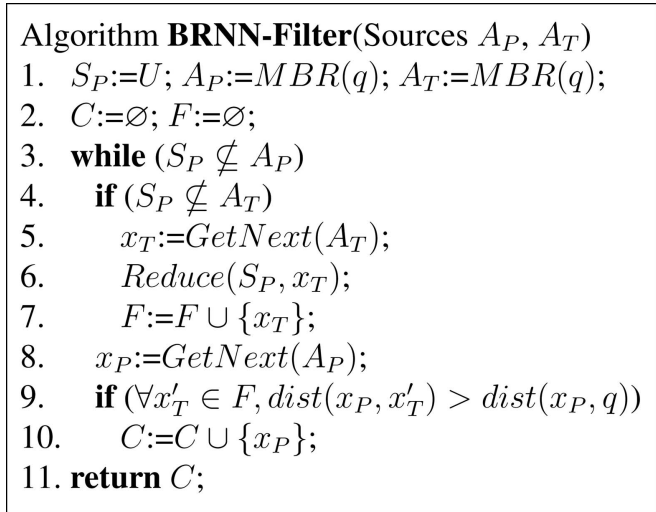

Fig. 11. Bichromatic RNN filter algorithm.

to each quadrant. Due to space constraints, the proof of the correctness of this approach is omitted. We stress that this technique is deterministic, as opposed to the probabilistic nature of selecting $k$-subsets in the TPL filter. In addition, it is applicable to any $L_{p}$ distance norms. The grid-based Greedy filter can also be easily extended for R $k$ NN search; a cell in this case is pruned if it falls outside $\perp_{q}(q, p)$ for at least $k$ points $p \in C \cup F$.

For the verification step of $\mathrm{R} k \mathrm{NN}$ search, for each candidate point $p$, we keep a counter of the points in $C \cup$ $F$ which are closer to $p$ than to $q$ during the filter step. Every time a new point is accessed, these counters are updated. Eventually, verification is required only for candidates for which the counter is smaller than $k$.

\subsection{Bichromatic RNN Search}

Bichromatic reverse nearest neighbor (BRNN) queries are a popular variant of RNN search. Given a point data set $P$, a site data set $T$, and a point $q$, the output of a BRNN query is $\{p \in P \mid \operatorname{dist}(p, q)<N N \operatorname{dist}(p, T)\}$, where $N N \operatorname{dist}(p, T)$ is the distance from $p$ to its NN in $T$. Stanoi et al. [26] first identified this problem and proposed an R-tree-based algorithm for it. An approximate Voronoi cell of $q$ with respect to a subset of points in the site data set is first computed. Then, a range query on the point data set is performed to retrieve some candidate points. Finally, candidate points are checked against the site data set to verify whether they are actual results.

Our BRNN algorithm also follows the filter-refinement framework. We first discuss the filter algorithm, which is shown in Fig. 11. $q$ is the query point, $P$ is the point data set, and $T$ is the site data set. $A_{P}$ and $A_{T}$ denote the accessed space of $P$ and $T$, respectively. $S_{P}$ represents the search space of $P$. A point worth noticing is that the candidate set $C$ only maintains the points in $P$ and the false-hits set $F$ only maintains the points in $T$. The algorithm synchronously accesses both data sets $P$ and $T$. This is a main difference from the algorithm in [26]. First, we retrieve a point from $T$, then reduce the search space $S_{P}$ and add the point to $F$. Second, we retrieve a point from $P$. It is added to the candidate set $C$ if it is nearer to $q$ than all the points in $F$. The algorithm terminates when $A_{P}$ contains $S_{P}$, meaning that all the candidates have been found.

Like the monochromatic RNN filter algorithm in Section 3.3, the above filter algorithm for the bichromatic case provides a generic framework for different filter methods. For the GetNext function (on either $A_{T}$ or $A_{P}$ ), we can use its implementations discussed in Section 4 . In the context of BRNN query, the objective of the Reduce function is to shrink the search space $S_{P}$ (for the point set $P$ ) by using a point $x_{T}$ from the site set $T$. Thus, all the three concrete filter methods discussed before can be applied for the Reduce function.

In the refinement step, for each candidate, we first determine its verification range and then issue a range search on the site data set $T$ in order to verify the candidate. As for simple RNN queries, concurrent verification is employed to improve performance (see Section 5.1).

\subsection{Searching without Sorted Accesses}

Consider a set of vertically partitioned data, distributed at different servers, accessible only by random accesses. For example, consider the same set of objects (e.g., restaurants) for which different information (e.g., ranking) is accessible at different Web servers (e.g., using the restaurant name as search key). In this section, we extend our RNN search techniques for the case where all data sources allow only random accesses. Notice that, even for the apparently simpler top- $k$ search problem, we are not aware of any existing work for the above scenario. For instance, although top- $k$ algorithms in [8], [9], [12] perform random accesses to data sources, they still require sorted accesses to be supported by at least one source.

Lemma 4 provides a lower bound for the required number of accesses to derive the RNN set. Its proof is straightforward since we cannot prune an object unless we have verified that it is outside the search space by checking whether there exists some dimension $i$ such that $p_{i}$ lies outside the range $\left[S_{i}^{-}, S_{i}^{+}\right]$. Therefore, we need to access at least one attribute of the object.

Lemma 4. Given that only random accesses to data sources are available, for an object $p$, at least one of its attributes must be accessed in order to decide whether $p$ lies outside the search space $S$.

Our proposed RNN algorithm relies on the formulation of the accessed space $A$ (see Section 3.1). However, the accessed space $A$ becomes undefined when sorted accesses are not allowed. Thus, we need to develop an alternative method that performs only random accesses to the data sources. Fig. 12 illustrates the pseudocodes of the filter and verification algorithms.

The filter algorithm consists of two phases. In the first phase (Lines 1-2), we perform one random access (of an attribute value) for each object in the data set. This is the essential cost of our RNN search (according to Lemma 4). Since we have no guidance on which dimensions to access first for each point, we perform these accesses in a roundrobin fashion (i.e., point $p^{i}$ is accessed at dimension $i$ modulo $d$ ). In this way, different attributes (of different objects) can be accessed in parallel. Another advantage of this approach is that the maximum load of all servers is minimized. In the second phase (Lines 3-16), we perform additional random accesses to the objects in order to compute the search space $S$ and store candidate objects in 


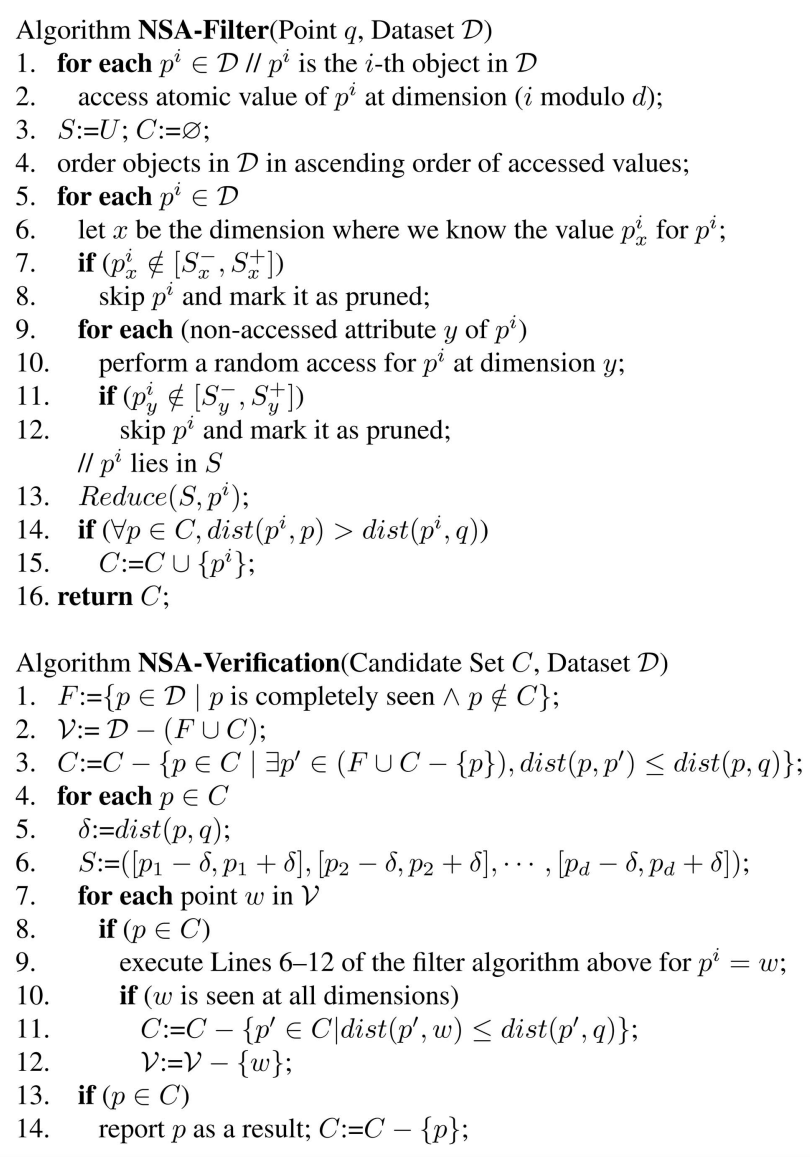

Fig. 12. RNN filter and verification algorithms using only random accesses.

$C$. We first set the search space $S$ to the universe $U$ and the candidate set $C$ to empty (Line 3 ). At Line 4 , objects are sorted in ascending order of their accessed values. This heuristic tends to tighten the search space earlier (in subsequent search); its effectiveness will be verified in our experiments. Then, for each point $p^{i}$ in the data set, we check whether it is definitely outside the search space $S$ (Line 7). If so, we skip processing $p^{i}$. Otherwise, we perform additional random accesses for $p^{i}$ (Lines 9-12) as long as $p$ has the potential to be located in $S$. If the point is found to be inside $S$, we can apply any filter technique in Section 4 for shrinking $S$. The point is added to $C$ if it is nearer to $q$ than to any of the points in $C$. It is worth noticing that the response time of the filter step can be reduced by parallelizing accesses for multiple candidates. In other words, while accessing the value of $p^{i}$ at dimension $y$ (Line 9) at the same time, we can access the values of (at most) $d-1$ other candidates at the $d-1$ dimensions different to $y$ (by picking candidates not seen in these dimensions).

The verification algorithm (NSA-Verification) operates in a similar way as the concurrent verification algorithm. For each candidate point $p$, we check whether it can be pruned by other points which have been seen in all dimensions. If $p$ remains a candidate, we check whether it can be pruned by any point in $\mathcal{V}$, the set of points not yet seen in all dimensions. For each object $w$ in $\mathcal{V}$, we perform accesses to its unknown values as long as it is possible for $p$ to be nearer to $w$ than $q$. If an object $w$ is seen at all dimensions, we check whether we can use it to prune any point in the candidate set (Line 11) and remove $w$ from $\mathcal{V}$ (Line 12). Finally, $p$ is reported as a result if it cannot be pruned by other points (Line 14). The same parallelization technique applied in the filter step can also be used during the verification, to minimize the response time.

\section{EXPERIMENTAL EVALUATION}

In this section, we evaluate the proposed RNN algorithms using synthetic and real data sets. All algorithms (TPL, G-IA for Greedy with intercept approximation, and G-HG for Greedy with hierarchical grid) were implemented in $\mathrm{C}++$. All experiments were performed on a Pentium $42.3 \mathrm{GHz} \mathrm{PC}$ with 512MB memory. The maximum recursion level of the search space reduction algorithm in G-HG is fixed to 5 (i.e., a grid of $32^{d}$ finest cells). For each experimental instance, the query cost is averaged over 100 queries with the same properties. We considered Euclidean distance in all experiments, since TPL is inapplicable for other distance metrics.

\subsection{Experimental Settings}

We generated uniform synthetic data sets (UI) by assigning random numbers to attribute values of objects independently. The default number of objects in a synthetic data set is $N=100 K$. We also used a real data set (JESTER [15]) which contains a total of $4.1 \mathrm{M}$ ratings of 100 jokes from $73 \mathrm{~K}$ users. A joke may not be rated by all users. We extracted the attributes (i.e., jokes) having value for at least $60 \mathrm{~K}$ objects (i.e., users) and then constructed binary tables for them (22 attributes). Query objects are users randomly chosen from the data set. For a particular query object $q$, we use only the attributes for which $q$ has ratings to issue a projected RNN query. In this way, we are able to extract query workloads with a specified number of query dimensions. The query result can be used to recommend $q$ to his/her RNNs as a potential "buddy" since $q$ has similar taste in jokes as them.

Attribute values of both the UI and JESTER data sets are normalized to the range $[0,1]$. We tried different access patterns for sequential accesses to the binary tables during RNN evaluation (i.e., round-robin, equi-depth, etc.). We found no practical difference between these schemes, thus we use a round-robin accessing scheme in all experiments reported here.

\subsection{Experimental Results}

\subsubsection{Monochromatic RNN Queries}

We study the performance of RNN search with respect to various factors. Fig. 13a shows the filter and verification costs (in terms of accesses) of the algorithms on the UI and JESTER data sets for queries with $d=3$ dimensions. The filter costs of the algorithms are proportional to their search space. The MBR clipping technique in TPL prunes the space too loosely. G-IA is more effective in space reduction than TPL. Finally, G-HG has the lowest filter cost as it utilizes the pruning power of discovered points in all quadrants. The concurrent verification algorithm is very efficient; verification costs less than 10 percent of the total cost. Since TPL 


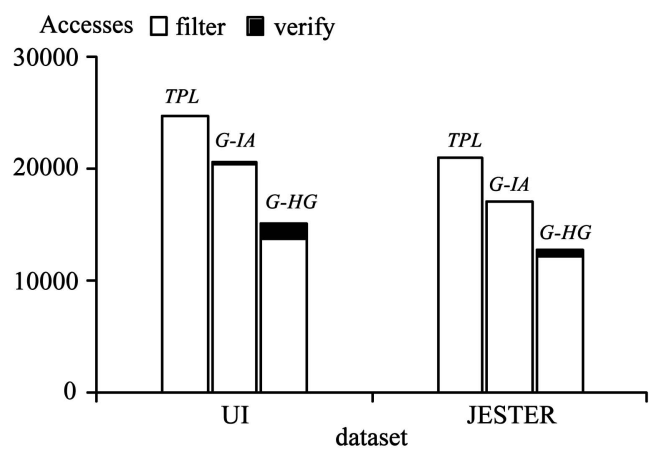

(a)

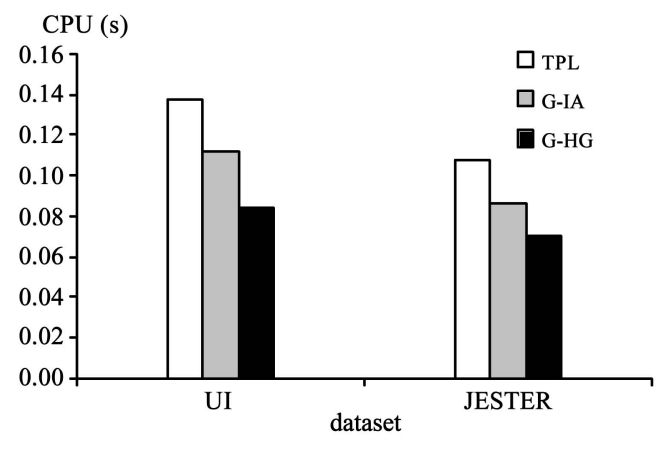

(b)

Fig. 13. Cost on different data sets, $d=3$. (a) Access cost. (b) CPU cost.

and G-IA search more space than G-HG, they eventually discover more points than G-HG, which can be used to prune more candidates. This explains the higher verification cost of G-HG compared to the other methods. As Fig. 13b shows, the CPU cost of the algorithms follows the same trend as the number of accesses. Unless otherwise stated, we consider JESTER as the default data set in subsequent experiments.

The next experiment justifies why we use only sorted accesses to the binary tables, whereas one could develop RNN algorithms that extend TA [11]. We implemented versions of TPL, G-IA, and G-HG that perform random accesses; whenever an object is seen from a binary table, $d-1$ random accesses to all other tables are applied to retrieve the values of the object in all other dimensions. Thus, there are no partially seen objects. Fig. 14 compares the original filter algorithms with their versions that employ random accesses (for queries with $d=3$ ). Observe that the total access cost when using random accesses is much higher than when not. In practice, their access cost difference is even higher, provided that random accesses are more expensive than sorted ones in real applications.

Fig. 15 shows the access and CPU cost of the algorithms as a function of query dimensionality $d$. G-HG outperforms the other algorithms in terms of accesses and the performance gap widens as $d$ increases. The pruning effectiveness of TPL and G-IA decreases with dimensionality. A bisector is less likely to prune all dimensions and reduce the global MBR, thus TPL is not very effective.

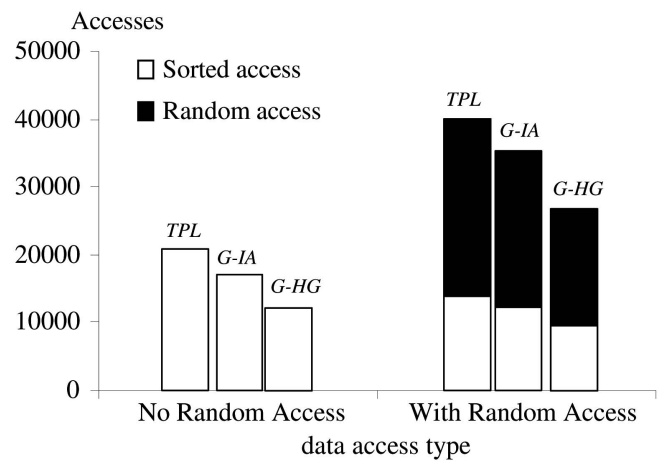

Fig. 14. Cost versus data access type, $d=3$, JESTER.
Besides, for a discovered point $p$, the number of neighbor quadrants increases with $d$ and G-IA fails to utilize $p$ in pruning them. The CPU cost has a slightly different trend. G-HG becomes very expensive at $d=5$ (and higher values) because it needs to examine a large number of hierarchical cells. We recommend G-IA for high query dimensionality because it achieves good balance between accesses and CPU cost.

Fig. 16 shows the cost of the algorithms as a function of the data size $N$, on 3D UI data sets. All of the algorithms are scalable as their costs increase sublinearly as $N$ increases. Again, G-HG outperforms the other methods and the performance gap widens as $N$ increases.

We also compared the algorithms for $\mathrm{R} k \mathrm{NN}$ search. Fig. 17 shows the performance of the algorithms with respect to $k$. Access costs of the algorithms increase sublinearly as $k$ increases. The cost of TPL increases at the fastest rate because it applies a heuristic, which only considers subsets of discovered points in reducing the search space. On the other hand, G-IA and G-HG employ deterministic and systematic approaches for reducing the search space effectively. Regarding CPU cost, TPL is the most expensive as it needs to examine several subsets of points. Also, G-HG becomes more expensive than G-IA at high values of $k$ because some high level (hierarchical) cells cannot be immediately pruned and more low level cells need to be visited.

\subsubsection{Advanced RNN Queries}

Fig. 18 shows the performance of the algorithms for bichromatic RNN queries with respect to the ratio of sites $T$ to points $P . T$ and $P$ are both 3D UI data sets and they have $200 \mathrm{~K}$ points in total. The result is similar to monochromatic queries; G-HG outperforms the other algorithms. When there are fewer sites and more points, the search space becomes larger and the density of the point data set increases. As a result, more verifications are needed.

Fig. 19a shows the progressiveness of the algorithms for a typical R4NN query on a 3D UI data set. All of the algorithms generate the first few results early because all of them follow the same filter framework algorithm. Their effectiveness in reducing the search space only affects their 


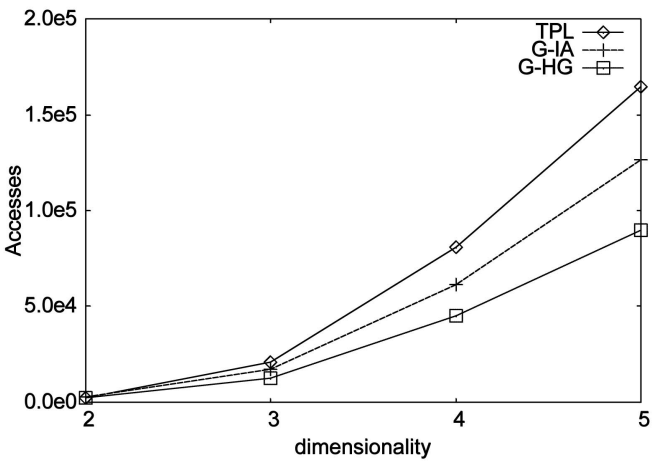

(a)

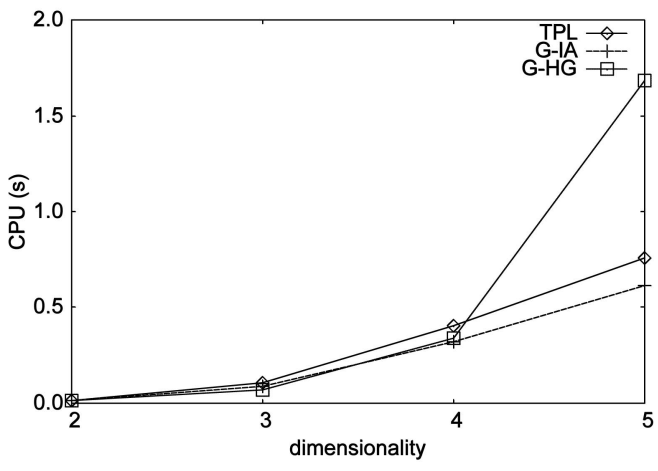

(b)

Fig. 15. Cost versus dimensionality $d$, JESTER. (a) Access cost. (b) CPU cost.

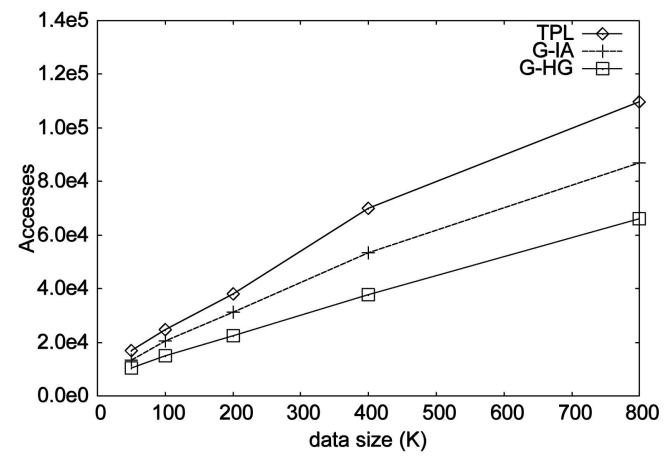

(a)

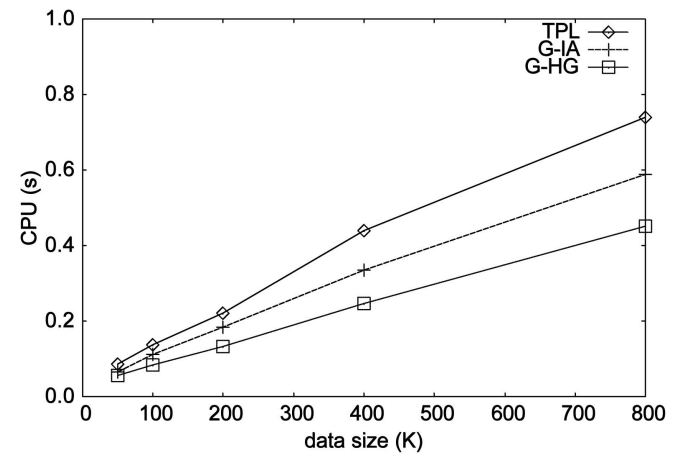

(b)

Fig. 16. Cost versus data size $N, d=3$, UI. (a) Access cost. (b) CPU cost.

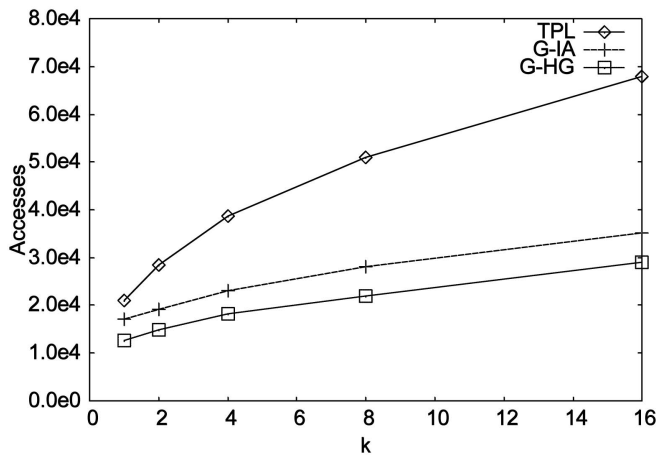

(a)

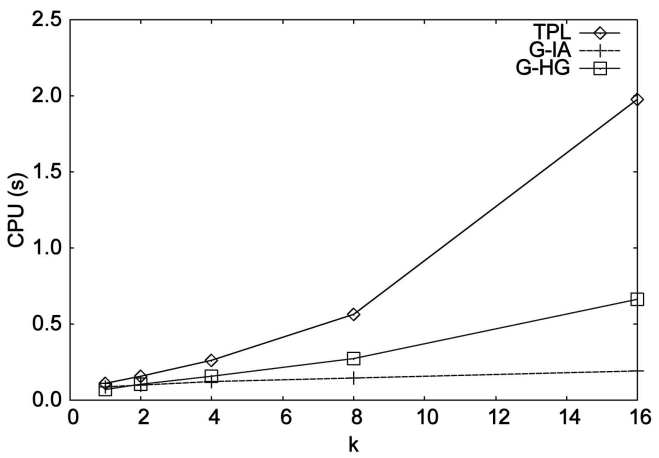

(b)

Fig. 17. Cost versus $k, d=3$, JESTER. (a) Access cost. (b) CPU cost.

total cost. The arrows indicate that G-HG terminated first, followed by G-IA and TPL. Fig. 19b shows the remaining access cost of G-HG estimated by the progress indicator onthe-fly for queries with different values of $k$. The initial cost estimate is around 2-3 times of the remaining cost. The estimated cost drops down fast to reasonable values after accessing a fraction of data values. At the end, the remaining access cost converges to 0 .

\subsubsection{Querying without Sorted Accesses}

The last set of experiments investigates the performance of RNN algorithms for the scenario where only random accesses to data sources (e.g., distributed Web servers) are allowed. The response time $^{3}$ of performing a random access to a data source is taken as one time unit. We assume that multiple servers can be accessed in parallel and count the total response time of each assessed method. In our comparison, we also include: 1) LB, which reflects the theoretical lower bound cost of retrieving RNN (see Lemma 4), and 2) UB, a brute-force

3. By response time here, we do not mean the CPU time, but we model the time required to stay connected and send requests to the servers in order to find the RNN set. We assume that all servers have the same access time, however, our methods can be adapted for the case where the assumption does not hold. 


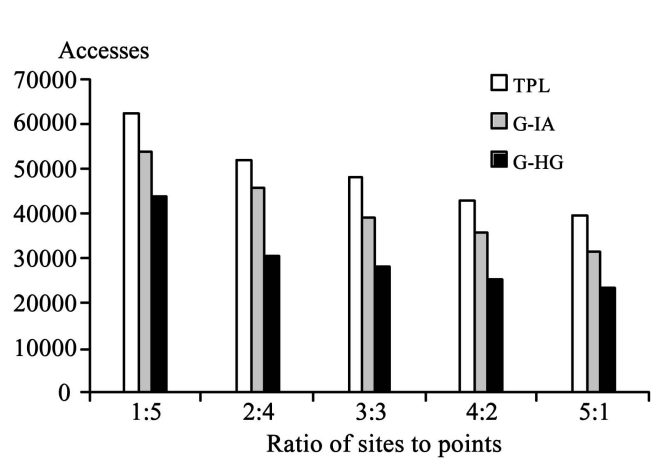

(a)

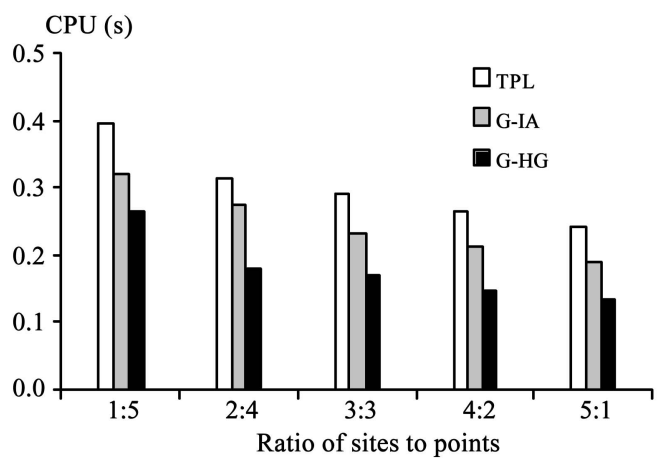

(b)

Fig. 18. Bichromatic RNN, cost versus site-to-point ratio, $|T|+|P|=200 K, d=3$, Ul. (a) Access cost. (b) CPU cost.

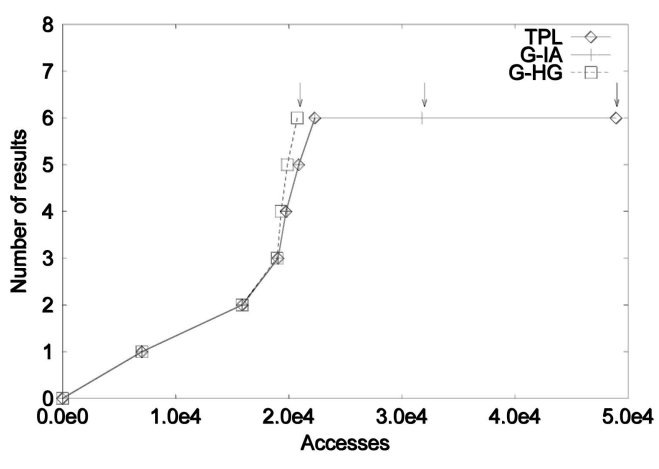

(a)

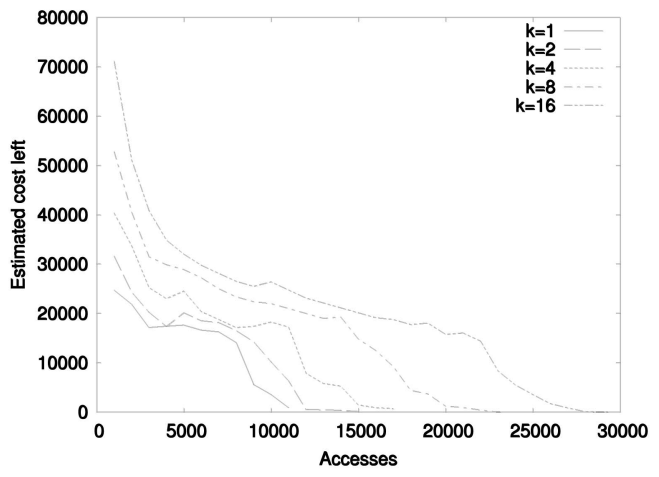

(b)

Fig. 19. Advanced RNN queries, $N=100 K, d=3$, Ul. (a) Progressive RNN, $k=4$. (b) Cost estimation for G-HG.

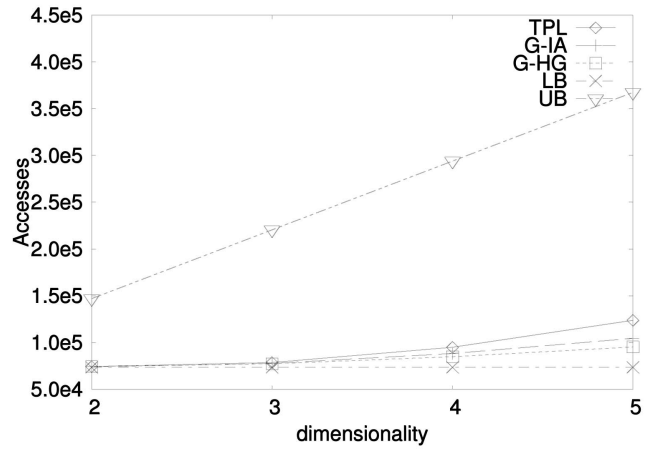

(a)

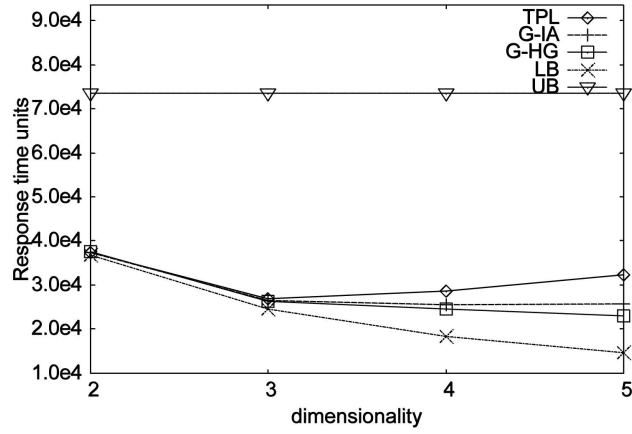

(b)

Fig. 20. Effect of $d, k=1$, random accesses only, JESTER. (a) Access cost. (b) Response time.

approach that performs parallel accesses to all attribute values for each object. Fig. 20 shows the cost of the algorithms as a function of query dimensionality $d$. Observe that the access cost of LB is very close to that of our algorithms. Again, G-HG outperforms its competitors. Unlike the experiment of Fig. 15, the algorithms are not much affected by the dimensionality curse. This is attributed to the fact that, after one to two attributes of an object have been accessed, the object is often found to be outside the search space and further accesses for the object can be saved. On the other hand, the response time of the algorithms follows a different trend due to two conflicting effects. First, when $d$ increases, accesses to more data sources can be parallelized and the response time is reduced. Second, the pruning effectiveness of the algorithms decreases with dimensionality and may lead to longer response time.

Fig. 21 shows the cost of the algorithms with respect to $k$. All of our algorithms have much lower access costs than UB. As $k$ increases, the access costs of G-IA and G-HG grow slowly. The response time of the algorithms follows the same trend as the access cost.

Finally, we study the effect of ordering the points in the second phase of the filter algorithm (Line 4 in Fig. 12). Fig. 22 plots the access costs of the algorithms for three different orders: 1) no order, 2) ascending order of the 


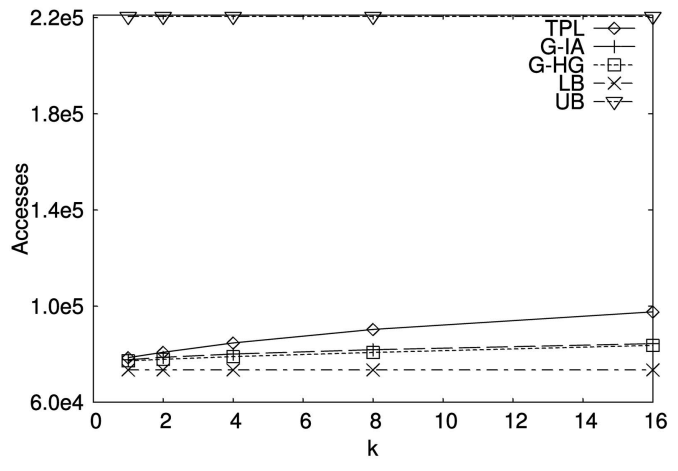

(a)

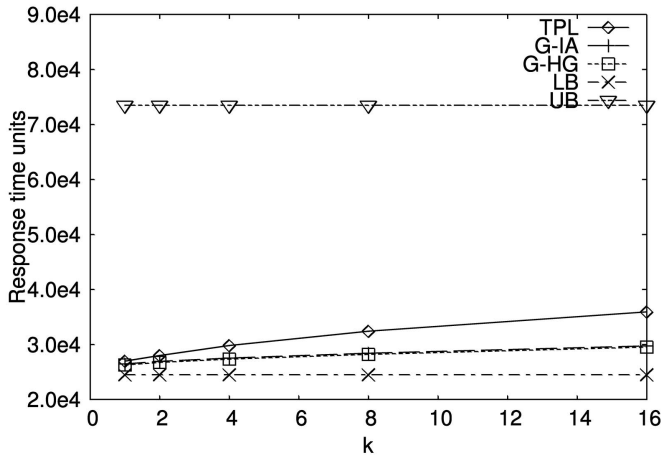

(b)

Fig. 21. Effect of $k, d=3$, random accesses only, JESTER. (a) Access cost. (b) Response time.

points by their accessed values (i.e., the default ordering), and 3) descending order of the points by their accessed values. The cost of LB (shown in a dotted line) also reflects the access cost in the first phase of the filter algorithm. Observe that the ascending order (i.e., the default order) minimizes the access cost. In particular, for all three methods, the access cost in the second phase of the filter step (i.e., the part above LB) is nearly halved from the worst order to the best order.

\section{Conclusion}

We proposed the first algorithms for projected RNN queries (and their variants) on the decomposed storage model and evaluated their performance on both synthetic and real data sets. We also proposed the first techniques for retrieving RNN results in a progressive way. Our techniques can efficiently process RNN queries in arbitrary-dimensional subspaces, at vertically partitioned distributed databases, and based on arbitrary distance measures.

Like most previous RNN methods, our proposal is based on a filter-refinement paradigm. We studied three alternatives for the filter step. Algorithm G-HG, which uses a grid to reduce the RNN search space, has been proved the most efficient in terms of data accesses, while G-IA, which approximates the space to be searched with the help of intercepts of perpendicular bisectors on the dimensional

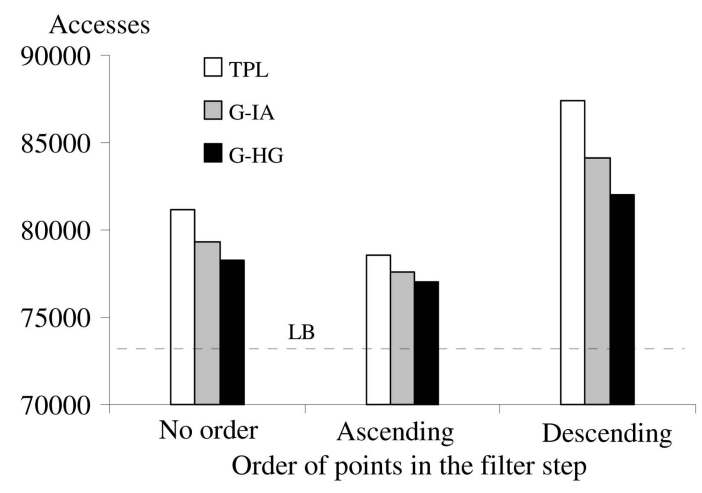

Fig. 22. Effect of ordering the points, $k=1, d=3$, random accesses only, JESTER. axes, has more balanced cost between accesses and computations. Finally, TPL, which is a direct extension of the best-known algorithm for indexed $L_{2}$ spaces, has the worst performance in all cases. In terms of flexibility, G-HG is applicable to any distance metric, G-IA is applicable to any $L_{p}$ distance norm, and TPL is only applicable to the $L_{2}$ norm. Finally, we optimized the verification step, with the introduction of a concurrent verification technique.

We also developed techniques for several variants of projected RNN search. We studied bichromatic and R $k \mathrm{NN}$ queries and showed how our methods can be adapted in this case. In addition, we solved the interesting problem of RNN retrieval when only random accesses to dimensional values of each object are allowed. We showed that our adapted RNN algorithms for this case require only marginally more accesses than the theoretical lower bound cost required to solve the problem.

In the future, we plan to extend our methods to the case of vertically distributed data at different servers, where the access cost/response time between servers varies. In this case, we can adapt the access patterns such that servers with faster response time are accessed more frequently. Finally, we will study the case where some sources allow sorted access and others allow only random ones.

\section{ACKNOWLEDGMENTS}

This work was supported by grant HKU 7380/02E from Hong Kong RGC. A preliminary version of this work appeared in [30].

\section{REFERENCES}

[1] Data Warehousing and OLAP: A Research-Oriented Bibliography, http://www.ondelette.com/OLAP/dwbib.html, 2006.

[2] Sysbase IQ White Papers, http://www.sybase.com, 2006.

[3] C.C. Aggarwal, C.M. Procopiuc, J.L. Wolf, P.S. Yu, and J.S. Park, "Fast Algorithms for Projected Clustering," Proc. 1999 ACM SIGMOD Int'l Conf. Management of Data, 1999.

[4] S. Arya and A. Vigneron, "Approximating a Voronoi Cell," HKUST Research Report HKUST-TCSC-2003-10, 2003.

[5] R. Benetis, C.S. Jensen, G. Karciauskas, and S. Saltenis, "Nearest Neighbor and Reverse Nearest Neighbor Queries for Moving Objects," Proc. Int'l Database Eng. and Applications Symp., 2002.

[6] K. Beyer, J. Goldstein, R. Ramakrishnan, and U. Shaft, "When Is 'Nearest Neighbor' Meaningful?," Proc. Int'l Conf. Database Theory (ICDT), 1999. 
[7] P. Boncz and M. Kersten, "MIL Primitives for Querying a Fragmented World," VLDB J., vol. 8, no. 2, pp. 101-119, 1999.

[8] N. Bruno, L. Gravano, and A. Marian, "Evaluating Top-k Queries over Web-Accessible Databases," Proc. Int'l Conf. Data Eng., 2002.

[9] K.C.-C. Chang and S. Hwang, "Minimal Probing: Supporting Expensive Predicates for Top-k Queries," Proc. ACM SIGMOD Conf., 2002.

[10] G. Copeland and S. Koshafian, "A Decomposition Storage Model," Proc. ACM SIGMOD Conf., 1985.

[11] R. Fagin, A. Lotem, and M. Naor, "Optimal Aggregation Algorithms for Middleware," Proc. 20th ACM SIGMOD-SIGACTSIGART Symp. Principles of Database Systems, 2001.

[12] R. Fagin, A. Lotem, and M. Naor, "Optimal Aggregation Algorithms for Middleware," J. Computer and System Sciences, vol. 66, no. 4, pp. 614-656, 2003.

[13] H. Ferhatosmanoglu, I. Stanoi, D. Agrawal, and A.E. Abbadi, "Constrained Nearest Neighbor Queries," Proc. Int'l Symp. Spatial and Temporal Databases, 2001.

[14] G.H. Gessert, "Four Valued Logic for Relational Database Systems," SIGMOD Record, vol. 19, no. 1, pp. 29-35, 1990.

[15] K. Goldberg, T. Roeder, D. Gupta, and C. Perkins, "Eigentaste: A Constant Time Collaborative Filtering Algorithm," Information Retrieval, vol. 4, no. 2, pp. 133-151, 2001.

[16] U. Güntzer, W.-T. Balke, and W. Kießling, "Towards Efficient Multi-Feature Queries in Heterogeneous Environments," Proc. IEEE Int'l Conf. Information Technology (ITCC), 2001.

[17] A. Hinneburg, C.C. Aggarwal, and D.A. Keim, "What Is the Nearest Neighbor in High Dimensional Spaces?" Proc. 26th Int'l Conf. Very Large Data Bases (VLDB), 2000.

[18] G.R. Hjaltason and H. Samet, "Distance Browsing in Spatial Databases," ACM Trans. Database Systems, vol. 24, no. 2, pp. 265318, 1999.

[19] F. Korn and S. Muthukrishnan, "Influence Sets Based on Reverse Nearest Neighbor Queries," Proc. ACM SIGMOD Conf., 2000.

[20] F. Korn, S. Muthukrishnan, and D. Srivastava, "Reverse Nearest Neighbor Aggregates over Data Streams," Proc. Int'l Conf. Very Large Data Bases (VLDB), 2002.

[21] G. Luo, J.F. Naughton, C. Ellmann, and M. Watzke, "Toward a Progress Indicator for Database Queries," Proc. ACM SIGMOD Conf., 2004.

[22] A. Okabe, B. Boots, K. Sugihara, and S. Chiu, Spatial Tessellations: Concepts and Applications of Voronoi Diagrams, second ed. Wiley, 2000.

[23] B.C. Ooi, C.H. Goh, and K.-L. Tan, "Fast High-Dimensional Data Search in Incomplete Databases," Proc. Int'l Conf. Very Large Data Bases (VLDB), 1998.

[24] A. Singh, H. Ferhatosmanoglu, and A.S. Tosun, "High Dimensional Reverse Nearest Neighbor Queries," Proc. Conf. Information and Knowledge Management (CIKM), 2003.

[25] I. Stanoi, D. Agrawal, and A.E. Abbadi, "Reverse Nearest Neighbor Queries for Dynamic Databases," Proc. ACM SIGMOD Workshop Research Issues in Data Mining and Knowledge Discovery, 2000.

[26] I. Stanoi, M. Riedewald, D. Agrawal, and A.E. Abbadi, "Discovery of Influence Sets in Frequently Updated Databases," Proc. Int'l Conf. Very Large Data Bases (VLDB), 2001.

[27] M. Stonebraker, D. Abadi, A. Batkin, X. Chen, M. Cherniak, M Ferreria, E. Lau, A. Lin, S. Madden, E. O'Neil, P. O'Neil, A. Rasin, N. Tran, and S. Zdonik, "C-Store: A Column-Oriented DBMS," Proc. Int'l Conf. Very Large Data Bases (VLDB), 2005.

[28] Y. Tao, D. Papadias, and X. Lian, "Reverse kNN Search in Arbitrary Dimensionality," Proc. Int'l Conf. Very Large Data Bases $(V L D B), 2004$.

[29] C. Yang and K.I. Lin, "An Index Structure for Efficient Reverse Nearest Neighbor Queries," Proc. Int'l Conf. Data Eng. (ICDE), 2001.

[30] M.L. Yiu and N. Mamoulis, "Reverse Nearest Neighbors Search in Ad-Hoc Subspaces," Proc. Int'l Conf. Data Eng. (ICDE), 2006.

[31] M.L. Yiu, D. Papadias, N. Mamoulis, and Y. Tao, "Reverse Nearest Neighbors in Large Graphs," Proc. Int'l Conf. Data Eng. (ICDE), 2005.

[32] J. Zhang, M. Zhu, D. Papadias, Y. Tao, and D. Lee, "LocationBased Spatial Queries," Proc. ACM SIGMOD Conf., 2003.

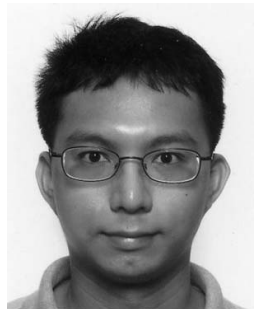

Man Lung Yiu received the bachelor's degree in computer engineering and the $\mathrm{PhD}$ degree in computer science from the University of Hong Kong in 2002 and 2006, respectively. $\mathrm{He}$ is currently an assistant professor in the Department of Computer Science, Aalborg University. His research interests include databases and data mining, especially advanced query processing and mining techniques for complex types of data.

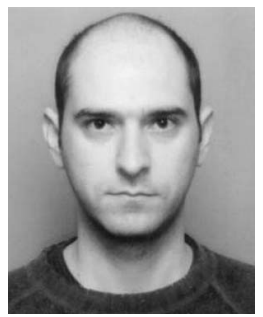

Nikos Mamoulis received the diploma in computer engineering and informatics in 1995 from the University of Patras, Greece, and the PhD degree in computer science in 2000 from the Hong Kong University of Science and Technology. Since September 2001, he has been an assistant professor in the Department of Computer Science at the University of Hong Kong. In the past, he has worked as a postdoctoral researcher at the Centrum voor Wiskunde en Informatica (CWI), The Netherlands. His research interests include complex data management, data mining, advanced indexing and query processing, and constraint satisfaction problems. He has published more than 60 articles in reputable international conferences and journals and served on the program committees of major database and data mining conferences.

$\triangleright$ For more information on this or any other computing topic, please visit our Digital Library at www.computer.org/publications/dlib. 